\title{
A Novel Robust Optimal Active Control of Vehicle Suspension Systems
}

\author{
Saber Fallah*, Aldo Sorniotti*, Patrick Gruber*, \\ *University of Surrey, Guildford, GU2 7XH \\ UK (Tel: 0044-1983-686528; e-mail:\{s.fallah, s.sorniotti, p.gruber\}@ surrey.ac.uk).
}

\begin{abstract}
Using Lyapunov theory, Pontryagin's minimum principle, and affine quadratic stability, a novel robust optimal control strategy is developed for active suspension systems to enhance vehicle ride comfort and handling performance. The controller has a simple structure, making its suitable for real-time implementation. The required sensor configuration includes a six-axis IMU and four LVDTs. The proposed controller is suitable for on-road commercial vehicles where ride comfort over bump disturbances and handling performance are the most concerns. The effectiveness of the controller is verified through simulation results using IPG CarMaker software.
\end{abstract}

\section{INTRODUCTION}

A suspension system of a vehicle mainly is designed so as to adequately support the vehicle weight, to provide effective isolation of the chassis against road irregularities, to maintain the wheels in appropriate position on the road surface and to keep tire contact with the ground. However, these objectives are in conflict due to requirements of the road holding and passenger comfort in wide range of road irregularities. For example, a soft damping is required to achieve superior ride quality at the expense of larger suspension deflection. In contrast, a large damping yields a better road-holding ability at the cost of comfort. While in primary vehicle suspensions, the geometric and dynamical properties of the suspension structure would be chosen by compromising some of those criteria, in modern suspension structures, a fully active or semi-active device is incorporated to meet these conflicting requirements (Fallah, S. et. al., [2009]).

Active and semi-active suspension systems have been the subject of research for more than two decades. However, this field of research is still open and automotive companies are actively looking for implementation of such systems on their commercial products. For instance, Mercedes-Benz has implemented an active suspension system, called active body control, on its SL, CL, and S class models (Streiter R. 2008).

There exist many control strategies for semi/active vehicle suspension application in the literature. However, optimal control is the most studied one. Linear Quadratic Regulator (Kumar et. al. [2006]), Linear Quadratic Gaussian (Sohn, et. el, [2004]), and Model Predictive Control (Göhrle, et. al., [2013]) are the most optimal controller investigated for vehicle suspension systems. Robust control techniques such as $\mathrm{H}_{\infty}$ (Fallah, et. al., [2012]), $\mathrm{H}_{2}$ (Hazell [2008]), and $\mathrm{H}_{\infty} / \mathrm{GH}_{2}$ (Akbari, et. al., [2010]) are other control strategies that have been investigated in the context of optimal control systems for the vehicle suspension systems. What makes the robust control popular in the context of the suspension control design is its ability to deal with frequency specifications and model uncertainties.

Usually, the ride comfort is quantified by vehicle body acceleration, whereas suspension stroke and tire deflection are considered for evaluation of vehicle handling and stability, respectively. Thus, the control objective typically has been defined so as to minimize a quadric function of acceleration, suspension stroke, tire deflection, and control signals. As another formulation, the control objective has been defined to minimize the body acceleration whereas suspension stroke, tire deflection and control signal are required to be kept within allowed bounds rather than to be minimized (Chen, et al., [2005] and Akbari, et al., [2008]).

Although the results presented in above-mentioned literature showed the effectiveness of the proposed controllers, the tire deflection and velocity have been considered as the states of the system. It is noted that measuring the tire deflection is very difficult or even impossible in practice. Although it is possible to employ an observer system to estimate the tire deflection, it causes difficulties in both implementation and control design.

This paper proposes a novel robust control architecture for vehicle suspension systems by defining a new objective control and a sensor configuration including one 6-axis Inertia Measurement Unit (IMU) and four Linear Variable Differential Transducers (LVDT).The rest of the paper is organized as follows. Section 2 explains the suspension modelling and system states configuration. Section 3 describes the control formulation while Section 4 represents the state estimation methodology. The effectiveness of the controller is investigated through simulation and is studied in Section 5. Finally, Section 6 summarizes the results of the work. 


\section{SYSTEM MODELLING}

The 2-DOF quarter-car model shown in Fig. 1 is used in this study. Despite of its simplicity, it represents the suspension dynamics with enough accuracy and captures major characteristics of the system.

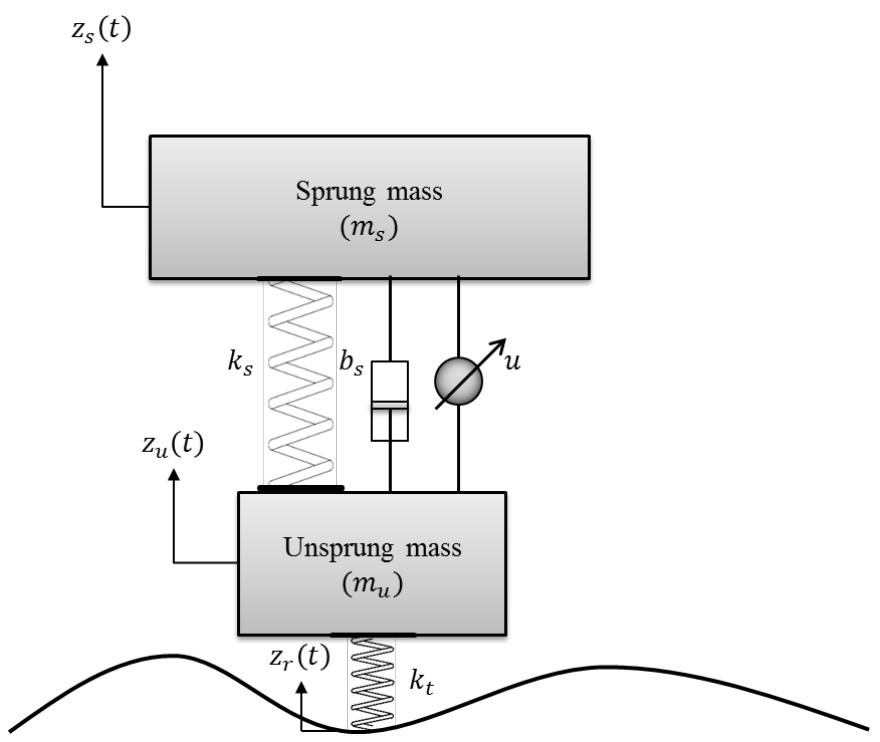

Fig. 1. active suspension system

In Fig. $1, z_{s}$ and $z_{u}$ represent the vertical displacement of the sprung and unsprung masses, respectively, while $z_{r}$ shows road irregularities. $k_{s}$ and $k_{t}$ indicate the suspension stiffness and tire stiffness, respectively. Also, $b_{s}$ and $u$ indicate the damping coefficient and active force, in order.

The equations of motion of the system are formulated as:

$\left\{\begin{array}{c}\ddot{z}_{s}=-\frac{b_{s}}{m_{s}}\left(\dot{z}_{s}-\dot{z}_{u}\right)-\frac{k_{s}}{m_{s}}\left(z_{s}-z_{u}\right)-\frac{1}{m_{s}} u \\ \ddot{z}_{s}-\ddot{z}_{u}=\frac{b_{s}}{m_{e}}\left(\dot{z}_{s}-\dot{z}_{u}\right)+\frac{k_{s}}{m_{e}}\left(z_{s}-z_{u}\right)- \\ \frac{k_{t}}{m_{u}}\left(z_{s}-z_{u}\right)+\frac{k_{t}}{m_{u}} z_{s}-\frac{k_{t}}{m_{u}} z_{r}+\frac{1}{m_{e}} u\end{array}\right.$

where $\frac{1}{m_{e}}=-\left[\frac{1}{m_{s}}+\frac{1}{m_{u}}\right]$.

This formulation allows defining the set of state variables as:

$$
x(t)=\left[z_{s}, \dot{z}_{s}, z_{s}-z_{u}, \dot{z}_{s}-\dot{z}_{u}\right]
$$

The state space description of the system is obtained as:

$$
\begin{gathered}
\dot{x}(t)=\left(\begin{array}{cccc}
0 & 1 & 0 & 0 \\
0 & 0 & -k_{s} / m_{s} & -b_{s} / m_{s} \\
0 & 0 & 0 & 1 \\
k_{t} / m_{u} & 0 & k_{s} / m_{e}-k_{t} / m_{u} & b_{s} / m_{e}
\end{array}\right) x(t) \\
+\left(\begin{array}{c}
0 \\
0 \\
0 \\
-k_{t} / m_{u}
\end{array}\right) w(t)+\left(\begin{array}{c}
0 \\
-1 / m_{s} \\
0 \\
1 / m_{e}
\end{array}\right) u(t)
\end{gathered}
$$

where the control input $u$ is defined as $f / f_{\max }$, with $f_{\max }$ being the actuator saturation force and $f$ actuator real force. $w=z_{r}$ is considered as road disturbance input.

\section{CONTROL FORMULATION}

In this section, based on the control methodologies proposed by (Lim et al. [2006] and Wu et al. [1996]), a suboptimal robust bang-bang control method is formulated for uncertain LTI systems such as active suspensions with actuator saturation, over the prescribed upper and lower bounds of structured real parameter uncertainties. The controller is derived through applying affine quadratic stability and minimizing the time derivative of affine Lyapunov function subjected to the limit of control force.

Considering state space equation of the form

$$
\dot{x}(t)=A(\theta) x(t) \quad x(0)=x_{0}
$$

where $\theta=\left(\theta_{1}, \theta_{2}, \ldots, \theta_{N}\right) \in R^{N}$ is a vector of uncertain parameters and $\theta_{i} \in\left[\theta_{i}, \overline{\theta_{i}}\right]$, where $\underline{\theta_{i}}$ and $\overline{\theta_{i}}$ are the lower and upper bounds for the uncertain parameter values. The matrix $A(\theta)$ is assumed to be stable and depends affinely on the parameters of $\theta_{i}$. That is,

$$
A(\theta)=A_{0}+\theta_{1} A_{1}+\theta_{1} A_{1}+\cdots+\theta_{N} A_{N}
$$

where, $A_{0}, A_{1}, A_{2}, \ldots, A_{N}$ are known fixed matrices.

The robust optimal bang- bang optimal controller is formulated as minimizing the time derivative of an affine Lyapunov function subjected to the limit of control force using the concept of the affine quadratic stability definition and multi-convexity concept (Eq. 5) in order to reduce the problem to linear matrix inequality (LMI) problem (Lim, et. al., [2006] and Gahinet, et. al., [1996]).

Consider a Lyapunov function defined as:

$$
V(x, \theta)=x^{T} P(\theta) x,
$$

where $P(\theta)$ is an affine function of $\theta$.

$$
P(\theta)=P_{0}+\theta_{1} P_{1}+\theta_{2} P_{2}+\cdots+\theta_{N} P_{N}
$$

Definition (Lim, et. al., [2006] and Gahinet, et. al., [1996]): With definition of Lyapunov function (6), the LTI system (4) with uncertain parameters $\theta$ is said to be affinely quadratically stable if there exist $N+1$ symmetric matrices $P_{0}, P_{1}, P_{2}, \ldots, P_{N}$ such that

$$
\begin{gathered}
P_{0}+\theta_{1} P_{1}+\theta_{2} P_{2}+\cdots+\theta_{N} P_{N}>0 \\
A(\theta)^{T} P(\theta)+P(\theta) A(\theta)<0
\end{gathered}
$$

hold for all admissible trajectories of the parameter vector $\theta$.

In other words, the definition states that $V(x, \theta)>0$ and $d V(x, \theta) / d t<0$ for all admissible parameter trajectories. It is worth mentioning that finding an affine Lyapunov matrix $P(\theta)$ can be turned into an LMI with variables $P_{0}, P_{1}, P_{2}, \ldots, P_{N}$.

In order to develop the control law, consider system (3) with uncertain parameter $\theta \in\left[m_{s}, \overline{m_{s}}\right]$ as the following uncertain LTI system:

$$
\dot{x}(t)=A(\theta) x(t)+B_{1} u(t)+B_{2} w(t), x(0)=0
$$
with force constraint

$$
|u(t)| \leq 1
$$

where $B_{1}$ is the control vector and $B_{2}$ is the disturbance vector. The time derivative of the Lyapunov function (6) for the system is: 
$\dot{V}(x, \theta)=x^{T}[A(\theta) P(\theta)+P(\theta) A(\theta)] x+2 x^{T} P(\theta) B_{1} u+$ $2 x^{T} P(\theta) B_{2} w$

Remark: The control law using (12) can be obtained using Pontryagin's minimal principle which states:

For a LTI system (10), there exists a nonzero vector $h$ such that

$$
h^{T} X^{-1}(t) B_{1} u^{*}(t)=\min _{u \in M}\left\{-h^{T} X^{-1}(t) B_{1} u\right\}
$$

for each time $0<t<t^{*}$. Here $X^{-1}(t)$ is the solution of the LTI and $u^{*}$ is the optimized control force.

According to Pontryagin's Minimum Principle, the optimal control force of minimizing this time derivative of parameterdependent Lyapunov function under the control force constraint (11) is in the form of:

$$
u^{*}(t)=-\operatorname{sgn}\left[B_{1}^{T} P(\theta) x(t)\right]
$$

where $P(\theta)$ satisfies the following equations which corresponds to $2^{N+1}+N$ LMI conditions:

$$
\begin{gathered}
P(\theta)>0 \\
A^{T}(\theta) P(\theta)+P(\theta) A(\theta)<0 \\
A_{i}^{T} P_{i}+P_{i} A_{i} \geq 0 \text { for } i=1 \text { and } 2
\end{gathered}
$$

In this study $N=1$ and therefore there are 5 LMI conditions to find $P(\theta)$.

The optimal actuator force for an active suspension system is in the form of:

$$
f^{*}=-f_{\max } \operatorname{sgn}\left[B_{1}^{T} P(\theta) x(t)\right]
$$

where $f^{*}$ represent the optimal actuator force. The developed control law act as a switch-controller. However, it is wellknown that switch control systems such as sliding mode controller introduces chatters to the system, leading to system unstability. Also, it is important to consider the rate of control signals due to physical nature of actuators. In order to address these issues, the discontinuous sgn-function-type control signal (17) is transformed to a continuous signal using sigmoid function for active suspension systems. The definition and characteristics of a sigmoid function is given in Appendix A. The modified control signal using sigmoid function is in the form of:

$$
f^{*}=-\frac{f_{\max }}{1+e^{\left(-a\left(B_{1}^{T} P x(t)\right)+c\right)}}
$$

where parameters $a$ and $c$ are design parameters. In addition, the proper adjustment of parameter $a$ allows to manage the rate of control signal.

\section{STATE ESTIMATION}

In current active suspension design, it is assumed that the vehicle includes a 6-axis IMU and a LVDT sensor is integrated to each suspension system. Thus, it is necessary to estimate the $z_{s}, \dot{z}_{s}$, and $\dot{z}_{s}-\dot{z}_{u}$.

It is possible to estimate the suspension velocity by direct differentiation of the suspension deflection signal. However, a low pass filter is required to avoid the amplitude amplification at higher frequencies. The main drawback of this approach is that a low pass filter introduces phase delay to the estimated signal thereby causing the reduction of the phase margin stability of the system. To avoid this drawback, the suspension velocity is obtained by differentiation of the suspension deflection signal using a hybrid-smooth derivative formula:

$$
\dot{\Delta L}=\frac{16 \Delta L_{0}+\Delta L_{1}+10 \Delta L_{2}-10 \Delta L_{3}-6 \Delta L_{4}+9 \Delta L_{5}}{28 \Delta t}
$$

where

$$
\Delta L_{i}=\Delta L(t-i) \quad i=0,1, \ldots, 5
$$

$\Delta L=z_{s}-z_{u}$ and $\Delta t$ is differential time increment.

The differentiator uses five previously measured data as well as the current one in order to estimate the derivative signal.

In order to estimate $\dot{z}_{s}$, first, the vehicle centre of gravity (C.G.) acceleration signal is integrated and the velocity of C.G. is obtained. For integration it is assumed that the acceleration is a zero mean signal. In addition, at each time instant the mean of velocity over integration interval time is subtracted from the calculated velocity signal. Then, the velocity of the C.G. is mapped to the each corner of the vehicle using the following relation.

$$
\begin{gathered}
\dot{z}_{\mathrm{s}}=\dot{z}_{C . G .}+(\vec{\omega} \times \vec{r}) \\
\vec{\omega}=\dot{\varphi} \vec{\imath}+\dot{\theta} \vec{\jmath} \\
\vec{r}=\left[\begin{array}{lr}
-a & b \\
-a & -b \\
w_{b}-a & b \\
w_{b}-a & -b
\end{array}\right]
\end{gathered}
$$

where, vector $\vec{\omega}$ represents the vector of angular rates of the vehicle body and vector $\vec{r}$ shows the position vector from C.G. to the each corner. $a$ is the distance between front axle and the C.G and $b$ is half of track width of the vehicle. $w_{b}$ stands for wheel-base of the vehicle. $\dot{\varphi}$ and $\dot{\theta}$ are the roll and pitch rates, respectively, and can be obtained from IMU sensor. It should be noted that the yaw rate is ignored in the abovementioned mapping since its effect is small. Finally, the state $z_{S}$ is estimated from integration of (21).

\section{CONTROLLER IMPLIMENTATION AND SIMULATION RESULTS}

In order to verify the effectiveness of the formulated control strategy, it is implemented on a virtual car in IPG CarMaker software. For the simulation purposes, it is assumed that the maximum control force is $6000 \mathrm{~N}$. It is also assumed that the front and rear sprung masses have $\pm 10 \%$ uncertainty around their nominal values. The simulation are performed under two driving scenario including bump disturbance with speed of 35 $\mathrm{km} / \mathrm{h}$ and Double-Lane-change (DLC). Fig. 2and Fig. 3 show the dynamic and kinematic properties of the candidate car in IPG CarMaker software.

For bump disturbances, the variations of vehicle body acceleration and displacement as well as the variations of body pitch angle and pitch rate are important in justification of vehicle ride comfort. Fig. 4 and Fig. 5 indicate these variations subject to road bump. As illustrated in these figures, the proposed active control system significantly reduces these variations while driving the states to the system equilibrium point faster compared to a passive system. Fig. 6 also shows the variation of active force for front-left and rearright suspensions. As shown, the force variations are bounded within the force defined boundaries. 
Roll angle and roll rates are the most important qualities to quantify the vehicle handling performance during manoeuvres. Fig. 7 represents the effectiveness of the controller in reduction of roll and roll rate variations during DLC manoeuvre. Also, the controller reduces the vehicle C.G. acceleration more than $50 \%$ compared to a passive vehicle system according to the results shown in Fig. 8. The trend of the active force variations are illustrated in Fig. 9.

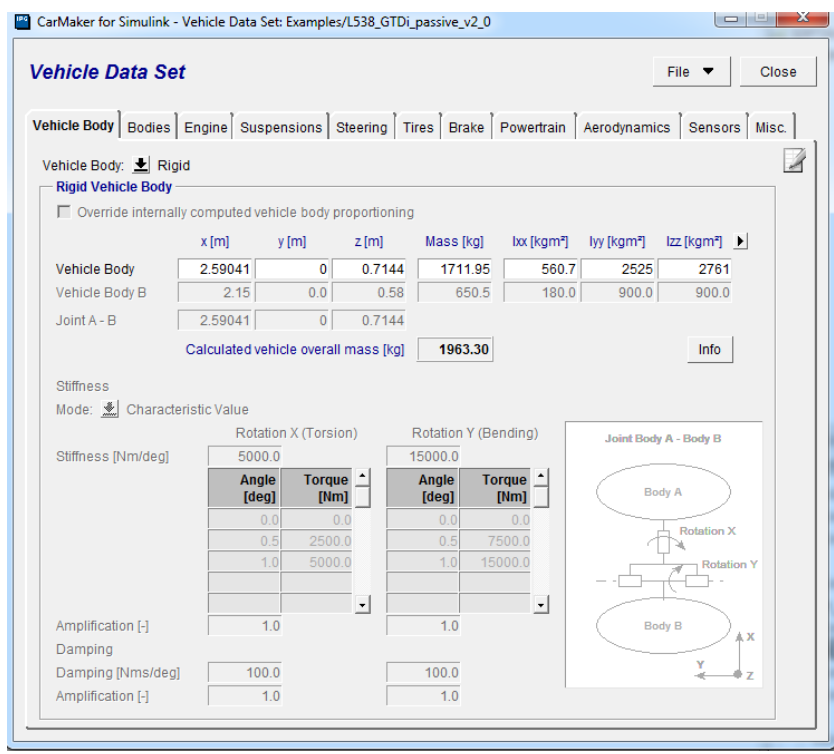

Fig. 2. Vehicle body dynamic and kinematic properties

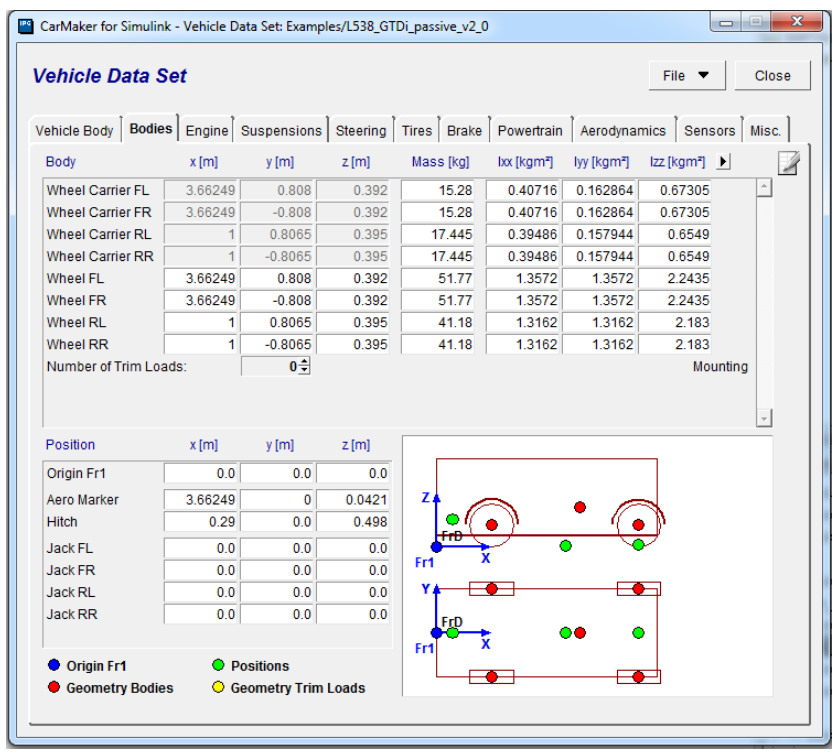

Fig. 3. Vehicle body dynamic and kinematic properties
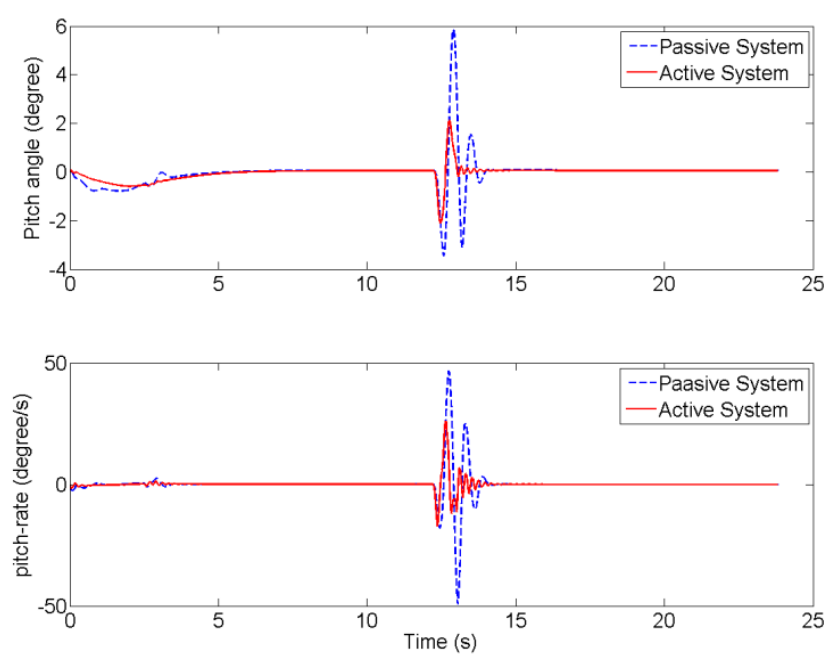

Fig. 4. Pitch and pitch rate variations subject to bump input
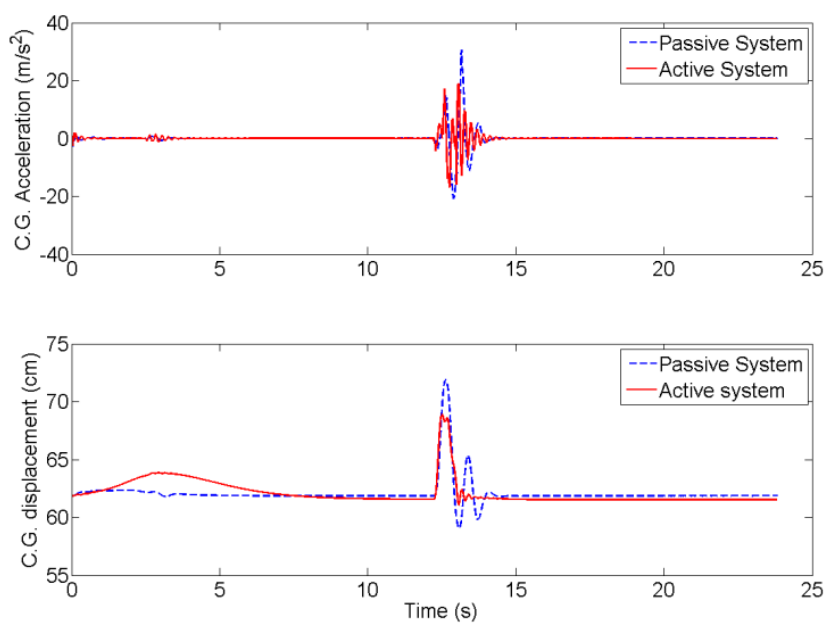

Fig. 5. Body acceleration and displacement subject to bump input
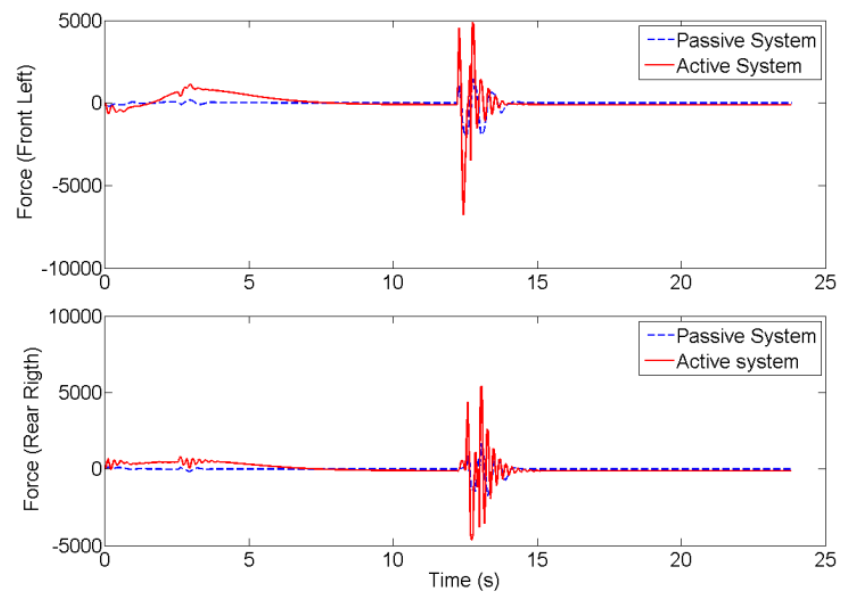

Fig. 6. Force variation subject to bump input 

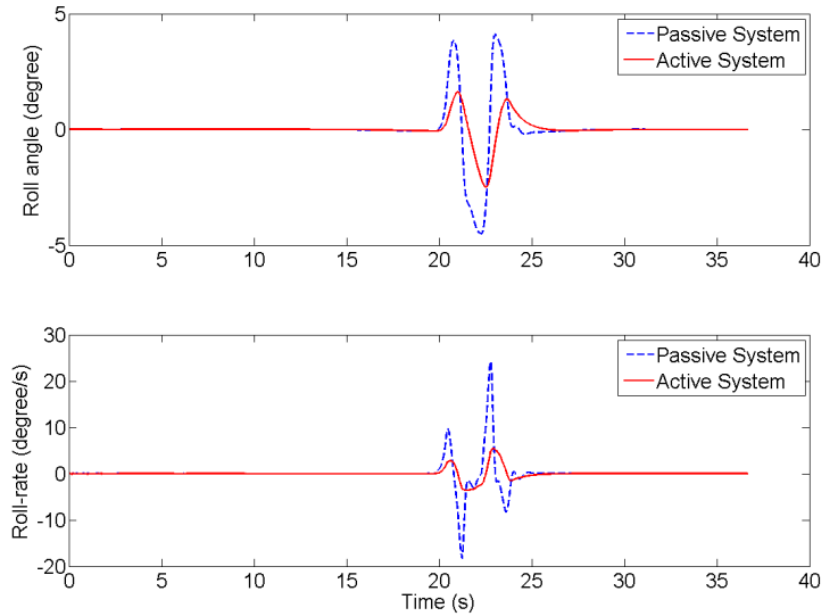

Fig. 7. Roll and roll rate variations in DLC manoeuvre

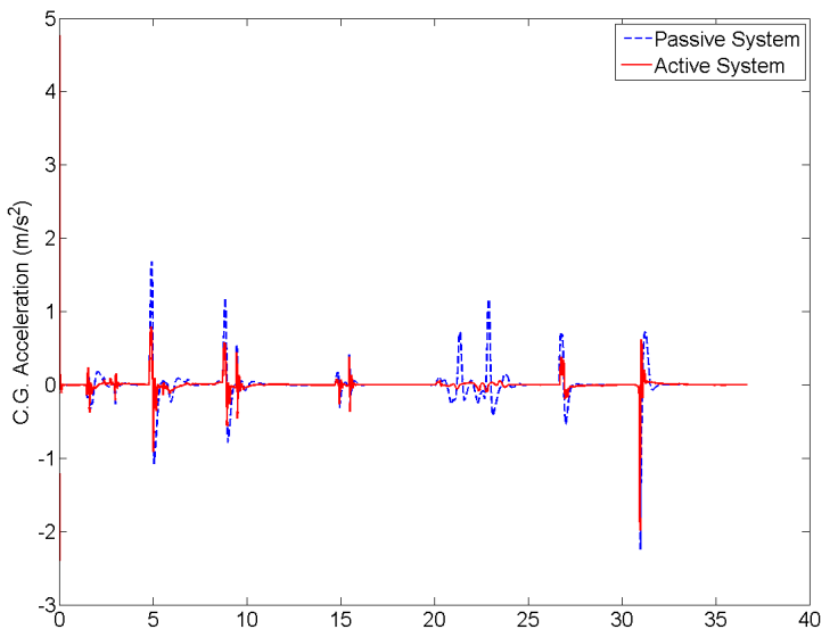

Fig. 8. Acceleration variation in DLC manoeuvre
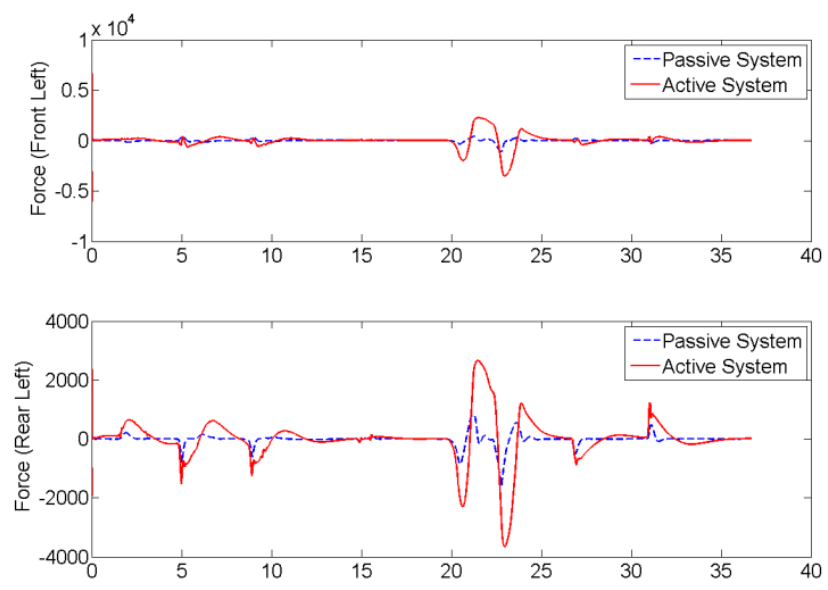

Fig. 9. Force variation in DLC manoeuvre

\section{CONCLUSION}

The paper proposed a novel robust bang-bang sub-optimal controller for active suspension systems. The objective of the controller is defined so as to drive the state of the system to its equilibrium point as quickly as possible while keeping the control effort at its pre-defined boundaries. The controller has a simple structure and easily implementable. The controller needs a sensor configuration including one 6-axis IMU and four LVDTs. The states of the system are estimated through a fifth-order differentiator and two integrators. The performance of the controller was verified through simulation results obtained by IPG CarMAker software. The controller is suitable for the commercial on-road vehicles where the bump disturbances in terms of ride comfort and vehicle handling performance are the main concerns.

\section{REFERENCES}

Akbari A. and Lohmann B. (2010), Output Feedback $\mathrm{H}_{\infty} / \mathrm{GH}_{2}$ Preview Control of Active Vehicle Suspensions: A Comparison Study of LQG Preview, Journal of Vehicle System Dynamics, Vol. 48, No. 12, pp. 1475-1494.

Akbari A. and Lohmann B. (2008), Multi-objective Preview Control of Active Vehicle Suspensions, Proceedings of the $17^{\text {th }}$ World Congress The International Federation of Automatic Control Seoul, Korea

Chen H., and Guo K. H., (2005), "Constrained Hळ control of active suspensions: An LMI approach," IEEE Trans. Control Syst. Technol., Vol. 13, No. 3, pp. 412-421.

Fallah, S., Bhat, R., Xie W. F., (2012), Optimized Control of Semiactive Suspension Systems Using $\mathrm{H}_{\infty}$ Robust Control Theory and Current Signal Estimation, IEEE Transactions on Mechatronics, Vol. 17, No. 4, pp. 767778.

Fallah, S., Bhat, R., and Xie, W. F., (2009), New Model and Simulation of Macpherson Suspension System for Ride Control Applications, Journal of Vehicle System Dynamics, Vol. 47, No. 2, pp. 195-220.

Gahinet P., Apkarian P., and Chilali M., (1996), Affine Parameter-Dependent Lyapanuv Functions and Real Parametric Uncertainty, IEEE Transactions on Automatic Control, Vol. 41, No. 3 pp. 436-442.

Göhrle C., Schindler A., Wagner A., and Oliver Sawodny, (2013), IEEE Transactions on Control Systems Technology, online edition.

Hazell A. (2008), Discrete-Time Optimal Preview Control, $\mathrm{PhD}$ Thesis, Imperial College University of London, Chapter 9.

Kumar M. S. and Vijayarangan S., (2006), Linear Quadratic Regulator Controller Design for Active Suspension System Subjected to Random Road Surfaces, Journal of Scientific \& Industrial Research, Vol. 65, pp. 213-226.

Lim C. W., Park Y. J., and Moon S. J., (2006), Robust Saturation Controller for Linear Time-invariant System with Structured Real Parameter Uncertainties, Journal of Sound and Vibration, Vol. 294, pp. 1-14.

Streiter R., 2008, Active Preview Suspension System ABC Prescan in the F700, ATZ worldwide, Vol. 110, No. 5, pp. 4-11.

Sohn H. C. and Hong K. T., (2004), An Adaptive LQG Control for Semi-active Suspension Systems, 
International Journal of Vehicle Design, Vol. 34, No. 4, pp. 309-325.

Wu Z. and Soong T. T., (1996), Modified Bang-bang Control Law for Structural Control Implementation, Journal of Engineering Mechanics, pp. 771- 777.

\section{Appendix A. FIRST APPENDIX}

The sigmoidal function, as given in the following equation, is a mapping on a vector $x$, and depends on two parameters $a$ and $c$.

$$
f(x)=\frac{1}{1+e^{-a(x+c)}}=\frac{1}{2}+\frac{1}{2} \tanh \left(\frac{1}{2} a(x+c)\right)
$$

where, $a$ shows the slope of the sigmoidal function and $c$ represents the center of the function. Depending on the sign of the parameter $a$, the sigmoidal membership function is inherently open to the right or to the left, and thus is appropriate for representing concepts such as "very large" or "very negative." More conventional-looking membership functions can be built by taking either the product or difference of two different sigmoidal membership functions. Figs AI and AII shows the sigmoid function response for different variables.

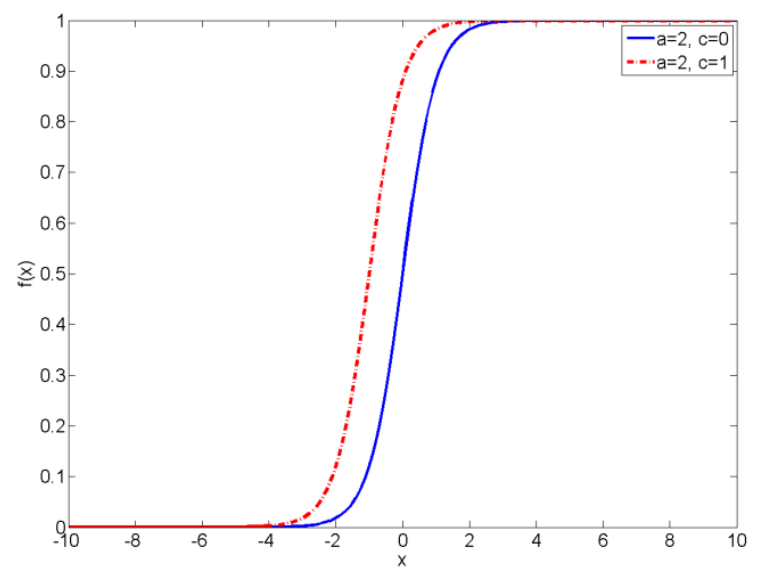

Fig. AI. Sigmoid function for $(a, c)=(2,0)$ and $(a, c)=$ $(2,1)$

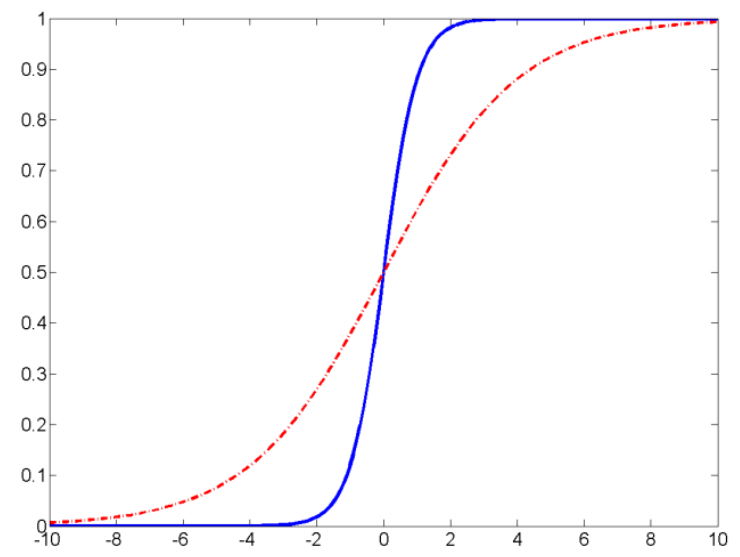

Fig. AII. Sigmoid function for $(a, c)=(2,0)$ and $(a, c)=$ $(0.5,0)$ 